\title{
A systematic literature review to understand cross-organizational relationship management and collaboration
}

\author{
Bruna Diirr \\ Federal University of the State of Rio de Janeiro \\ (UNIRIO) \\ bruna.diirr@uniriotec.br
}

\author{
Claudia Cappelli \\ Federal University of the State of Rio de Janeiro \\ (UNIRIO) \\ claudia.cappelli@uniriotec.br
}

\begin{abstract}
An increasingly dynamic, unpredictable and challenging environment leads organizations to cross their own borders and establish partnerships to other organizations for remaining competitive. This crossorganizational relationship allows participating organizations to share resources with each other and collaborate to better handle an identified opportunity for joint work. However, besides having a mutual or compatible goal, it is common that these organizations face several challenges during the partnership. The present research aims to explore the crossorganizational relationship management. To this end, this paper outlines the systematic literature review performed to understand the collaboration and relationship establishment between different organizations and organize an ICT related body of knowledge about the topic. A discussion about the findings, challenges and open issues identified from the retrieved literature is also provided to guide further work.
\end{abstract}

\section{Introduction}

Globalization, economic fluctuation, technological innovations, fast dissemination of information, the need for improving internal processes and reducing time and cost of product development, the urge for adding value to their own business, responding to market and costumers' demands or providing outcomes for Society's common good through transparency of processes and information, have led to organizations' change and adaptation. A usually adopted approach to face this dynamic and unpredictable environment have been crossing organizational borders and establishing partnerships with other organizations, which may be their rivals or operate in businesses than their own [1][2][3][4][5][6][7].

When investing in a cross-organizational relationship, organizations aim to combine resources, knowledge and/or power in benefit of participating organizations, and share the results achieved from the partnership [8]. These organizations select a specific type of relationship, considering the identified opportunity for joint work, faced reality and established agreements, which guides how collaboration will occur to achieve a common goal. Within a partnership, they can share existing human and financial resources, systems, equipment, work processes, skills and information, thus having access to a wider range of tools at a more favorable cost than they would on their own. In addition, they can organize information that is often related to the same business process, but as each part of this process is performed by a different organization, it is distributed in isolated databases. Besides that, these organizations can handle new business opportunities, respond to market challenges and costumers' demands, and deliver products and services more quickly and with more quality by complementing existing skills. Moreover, they will share all the risks, responsibilities and benefits arising from the partnership [5][7][8][9][10][11][12].

Despite all these benefits of cross-organizational relationships, participating organizations may face several challenges. Cross-organizational relationships stimulate the interaction of organizations with different characteristics, cultures and values. It increases the probability of facing misunderstandings and conflicts, thus influencing the partnership performance alignment. Organizations should develop skills to work in this new dynamic by identifying, integrating and managing all shared elements to ensure that these elements favor the execution of activities supporting the group strategy integration and mutual goals achievement. If it is not possible to establish a compromise between all existing dynamics, the crossorganizational relationship may fail, even leading to the partnership dissolution [1][7][8][13][14][15][16].

The present research aims to explore the challenge of managing cross-organizational relationships. As a broad and not well-structured topic, a systematic literature review was performed to retrieve what has been investigated on cross-organizational relationship 
management in the last decade. It allows us to understand how literature has been facing collaboration and relationship establishment between different organizations, besides organizing an ICT body of knowledge about this topic. The analysis of existing proposals, main challenges and open issues on crossorganizational relationship management provides inputs for further research on making organizations involved in cross-organizational relationships more integrated, prepared to interoperate their processes and information, and able to develop skills to act and achieve mutual goals even with existing differences.

The paper is organized as follows: Section 2 introduces the systematic literature review. Section 3 details the designed protocol for the systematic review on cross-organizational relationship management and obtained results from its execution. Section 4 discusses the findings, main challenges and open issues identified from the retrieved literature. Finally, Section 5 concludes the paper.

\section{Systematic literature review}

A systematic literature review (SLR) uses a welldefined methodology to identify, analyze and interpret all available research relevant to a specific question, area or phenomenon of interest, providing ways to perform comprehensive, unbiased and repeatable literature analysis. It aims to present a fair evaluation of a topic by using a trustworthy, rigorous and auditable methodology, becoming a powerful tool to collect and structure knowledge in a systematic and reproducible way [17][18]. SLRs are well known in disciplines such as Medicine and the interest in this methodology has been increasing in other areas, such as empirical software engineering [18][19].

The main reason for undertaking SLRs is to increase the material quality about an investigated topic. It guides the research process, positions the researcher in different areas and approaches on the research question, allows to assess the undertaken efforts and avoids overlapping efforts. The SLR helps to summarize empirical evidence of benefits and limitations of a specific topic, to identify gaps in current research to suggest areas for further investigation, to provide a framework to appropriately position new research activities, examine the extent to which empirical evidence supports/contradicts theoretical hypotheses or even assist the generation of new hypotheses. However, conducting an SLR requires considerably more effort than traditional literature reviews since it aims an in-depth and comprehensive investigation of an area of interest [17][18].

Most of SLRs follow the three-phase process defined by Kitchenham [17] (Figure 1). During planning, the researcher identifies the need for review, specifies the research question(s), develops the review protocol and evaluates it. During execution, researcher performs searches in defined search engines, assesses the retrieved studies using the established criteria, and extracts and synthesizes relevant data from selected studies. During reporting, the SLR results are documented and published.

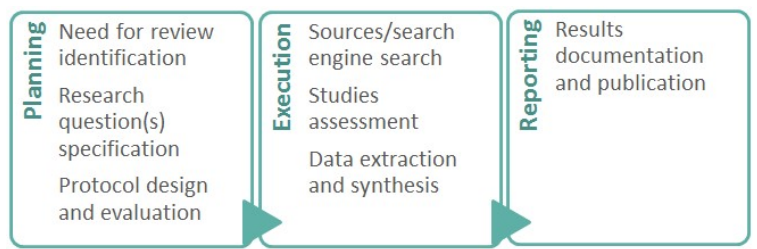

Figure 1. SLR process

\section{SLR on cross-organizational relation- ship management}

The SLR on cross-organizational relationship management has followed the steps showed in Figure 1. During planning, researchers have identified the need for an SLR, since the initial study on this broad topic revealed a huge number of related material, but no systematization has been identified to understand the collaboration and relationship establishment between different organizations and organize an ICT related body of knowledge about this topic. After that, they have developed the review protocol ${ }^{1}$ based on the protocol defined by Biolchini et al [20]. Our SLR protocol specifies the research question (What have been discussed and developed in the context of cross-organizational relationships management in the last decade?), sources (Compendex, IEEEXplore, Web of Knowledge and Scopes) and criteria for studies inclusion (publications describing difficulties, solutions, limitations and challenges of crossorganizational relationships management; relevant studies cited in retrieved studies) or exclusion (old studies; similar studies from other selected studies). The PICO approach was selected for research question definition, which structures this question into four elements [21]: population, the group that will be observed by the intervention (research, projects, initiatives and discussions in cross-organizational relationships management); intervention, what is going to be observed in the context of the planned SLR (proposals in business process modeling, organizational architecture, knowledge management, transparency, collaboration and information systems); comparison, a baseline or initial data set that researcher already possesses (no initial data set for our

${ }^{1}$ Available at https://goo.gl/w51CNz 
SLR); outcome, the results expected in the end of SLR (identification of difficulties faced, existing proposals limitations and research challenges). This structure has helped to develop the search string that was applied to the defined search engines, following the template "Population AND Intervention AND Comparison AND Outcomes"2:

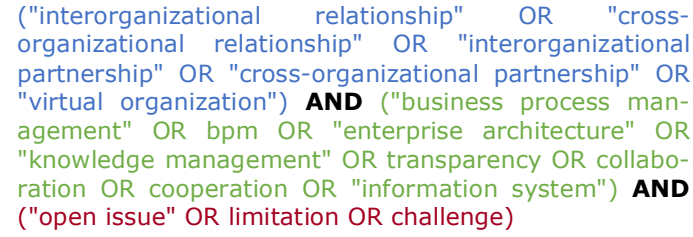

After planning, researchers have run the search string in each defined search engines, which has returned a total of 314 papers. All retrieved studies were imported into JabRef. This reference manager tool has helped the filtering process, which consists of four steps: duplicates elimination since studies may be indexed by different search engines; title and abstract filter, that excludes studies, which clearly dealt with irrelevant topics to this research based on the exclusion criteria; full-text availability filter since not all papers were available for download; and $p a-$ per reading filter, when researchers assess the remaining papers based on the established inclusion and exclusion criteria. JabRef has also allowed the creation of custom fields for storing extracted data and tracking decisions at each stage of the filtering process. After the filtering process, 30 papers were selected. Table 1 shows the execution phase results ${ }^{3}$.

Table 1. SLR execution results

\begin{tabular}{|c|c|}
\hline Retrieved papers & 314 \\
\hline After duplicates elimination & 207 \\
\hline After title/abstract filter & 68 \\
\hline After full-text availability filter & 53 \\
\hline After paper reading filter & 30 \\
\hline
\end{tabular}

\section{Cross-organizational management overview}

relationship

Researchers have consolidated relevant information from the 30 remaining studies into a mind map ${ }^{4}$, which is a powerful tool to visually organize material about a topic that is not well understood or structured. The mind map development adopted an approach showed in Figure 2. The top-down analysis was based on the way that remaining studies have

\footnotetext{
${ }^{2}$ As there was no initial data set for comparison in the designed protocol, it is not shown in search string.

${ }^{3}$ Reference information available at https://goo.gl/e46wxU

${ }^{4}$ Full version available at https://goo.gl/tLncJK
}

introduced the cross-organizational relationships management to identify a set of candidate categories (e.g., many papers described motivations, definitions, relationship types, benefits, challenges). After this, the bottom-up analysis aimed to refine the candidate categories based on topics that were discussed by papers in a less structured way (partnership structure, life cycle, mechanisms for analysis, researched areas) to validate if candidate categories could group the largest number of topics addressed by papers, besides identifying missing categories. The final categories were used as the first level of the mind map, from which additional information can be accessed. Following subsections details the SLR findings.

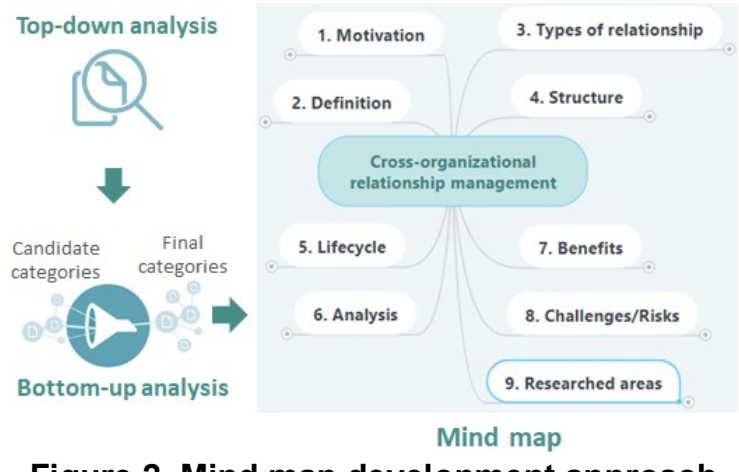

Figure 2. Mind map development approach

\subsection{Motivation and definition}

As mentioned before, different reasons may lead organizations to cross their own borders and start collaborating with other organizations. Environment dynamicity and unpredictability; economic fluctuation; globalization; technological innovations; fast dissemination of information; the need for improving internal processes/services/systems and for reducing time and cost of product development; the urge for adding value to their own business and responding to market and costumers' demands; the need for innovation and facing an environment with competition no longer between organizations, but between organizations networks; increasingly demand for transparency in existing processes and information; facing challenging opportunities that cannot be handled by their own etc. [1][7][10][11][22][23][24][25].

What we call cross-organizational collaboration may be defined as a process through which organizations, which see different aspects of a same problem, can constructively explore their differences, exchange skills/competencies/expertise, change activities, share their resources, enhance capacity for mutual benefit and search for solutions that go beyond their own limited vision of what is possible to do to achieve a mutual goal [26][27][28][29]. 


\subsection{Types of relationship}

The cross-organizational collaboration may be established through different types of relationships, which may occur in an ad hoc or a more structured way. Ad hoc collaboration occurs when organizations interact with each other in an informal way and without a certain regularity. A more structured collaboration may vary from a weak collaboration, when one organization has more power than the other(s) (i.e. mergers, acquisition, outsourcing, distribution, licensing, franchise etc.), to a strong collaboration, when organizations have certain power equality (i.e. consortium, alliances, networks, joint ventures, cooperatives, virtual enterprise etc.) [5][8][30][31][32].

Choosing the most appropriate relationship to the reality faced by organizations intending to establish a partnership considers: the goals to be achieved and common interests; the number, multidisciplinarity and heterogeneity of participating organizations; the environment and relationship dynamicity; trust arising from the transparency between those involved; the cooperation scope, being limited to a specific business unit or to the organization as a whole; the power ratio, with one organization concentrating all power or organizations having power equality; the cooperation type, occurring unilaterally or bilaterally; the relationship duration, being restricted to a specific project or regularly occurring through business processes; the communication structure, with information exchange occurring in an integrated or informal way; decisions autonomy, with organizations being independent during decision-making or all decisions occurring centrally; risk, rewards and responsibilities sharing, occurring unilaterally or bilaterally; the relationship formalization and agreement, being based on trust or legally constituted [5][7][11][15] $[22][28][30][33][34][35][36][37][38][39]$.

\subsection{Structure}

The chosen relationship type also influences the cross-organizational structure. Different collaboration patterns may be found. Arasteh et al [40] discuss that three possible topologies for cross-organizational relationships are found in literature: supply-chain, when each participant organization interacts with its direct neighbor in a linear interaction; star or hub, when a central organization manages the interaction between the different participant organizations; and peer to peer, when interaction between participant organizations does not follow a hierarchy and collaboration can be of different shapes and forms.

Van Fenema et al [7] propose a taxonomy that categorizes cross-organizational relationships into three possible structures: horizontal, when different organizations are part of similar value chains, geographic areas or work context, share resources and processes to better serve their customers demands, reduce costs and improve innovation and service quality; vertical, when an organization rearranges its services and business processes in a customersupplier relationship to better use the partnership capabilities and resources; hybrid, when organizations engage in a both horizontal and vertical cooperation, being involved in a complex network of stakeholders to achieve a mutual goal.

It is important to highlight that these collaboration patterns may be applied to how participating organizations manages the cross-organizational relationship and/or interacts with each other. For instance, it is possible that the cross-organizational management follows a star topology, but the interaction between organizations resembles a peer to peer topology [40].

\subsection{Lifecycle}

Literature details several proposals to characterize the cross-organizational relationship dynamics, from its establishment until its finalization [7][22][25][33] [40][41][42]. Based on these proposals, it is possible to say that the cross-organizational relationship life cycle has the five phases shown in Figure 3.

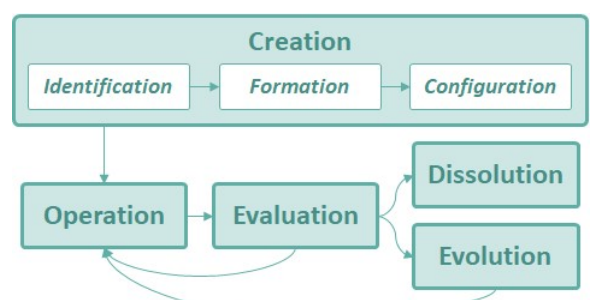

\section{Figure 3. Cross-organizational relationship lifecycle}

During creation, the cross-organizational relationship is shaped, following three steps: identification, when the business opportunity is characterized and goals are identified; formation, when possible partners are identified, evaluated and selected based on the mutual goals; and configuration, when participating organizations detail both organizational and cross-organizational business model, plan how collaboration and operation should occur, establish performance indicators, negotiate and analyze risks and establish a formal or informal agreement. After that, operation starts and organizations share their resources and perform actions to achieve the mutual goals. Organizations should also evaluate their own and the cross-organizational performance to guarantee that the partnership is achieving the desired re- 
sults. From the evaluation, they may decide for an evolution, when partnership adjustments are made, leading to a new form of collaboration and/or purpose; or the partnership dissolution, which occurs when the goal is achieved or due to relationship problems. It is necessary to determine the collaboration inheritance and finish legal issues.

\subsection{Analysis}

Caroll and Wang [26] argue that organizations are social-technical systems, i.e. a result of the linking between the available social and technological tools. Besides that, they claim that organizations should be understood contingently, since they are shaped by their histories and futures, and socially, as they are a collection of actors with individual goals and abilities, shared visions and common ground, mutual conflicts, feelings of trust/belonging/commitment, beliefs about personal/collective efficacy, and varying capacities and motivations for collaboration. All this influence and should be considered during crossorganizational analysis (Figure 4).

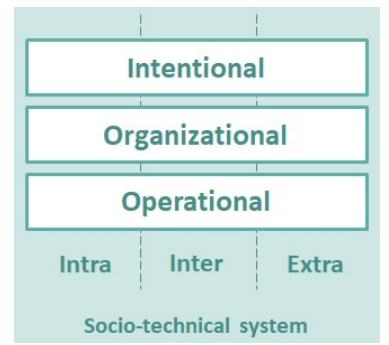

\section{Figure 4. Cross-organizational relationship analysis (based on [26] and [30])}

Priego-Roche et al [30] proposes that the crossorganizational analysis should consider three perspectives: intra-organizational, which focuses on analyzing each organization in terms of interaction between administrative and production structures, existing business processes and relevant and useful resources to the partnership; interorganizational, which focuses on analyzing the relationship between organizations to determine and assess the joint work compromises and mutual goal achievement; and $\boldsymbol{e x}$ tra-organizational, which focuses on analyzing the relation between cross-organizational relationship and its external environment. Besides that, each perspective analysis should be applied to the following three levels: intentional, comprising opportunity and partner identification, collaboration willingness, goals definition etc.; organizational, comprising business model definition, collaboration and operation planning, performance indicators establishment etc.; and operational, comprising operational process detailing and technological resources organization, such as information systems and data stores.

\subsection{Benefits and challenges}

Several benefits arise from cross-organizational relationships. They allow organizations to handle new business, market and technology opportunities; increase business networking; respond to market challenges and costumers' demands, capture resources and deliver products/ services with more agility and quality; have access to a wider range of tools (human and financial resources, systems, equipment, work processes, information etc.) at a more favorable cost; complement existing skills and knowledge; share all the risks, responsibilities and benefits arising from the partnership; innovate and provide new products/services etc. [5][7][8][9][10][11][12].

However, making organizations involved in cross-organizational relationships being integrated and developing skills to collaborate even with their differences is not a trivial task. Organizations should be prepared to handle several challenges so that joint work is effective, mutual goals are achieved and the relationship can be considered effective and successful [13][14][16]. These challenges may be classified into external challenges, which comprises environment challenges, such as natural, political, social, economic risks; internal challenges, which comprises intra-organizational challenges, such as strikes, production issues, infrastructure problems etc.; and network related challenges, which comprises challenges inherent to interaction between organizations, such as management, business processes, knowledge management and collaboration issues [43].

Management challenges are those related to how organizations manage the partnership, such as partners' identification and selection; planning process optimization; analysis and coordination of different organizations; management of new work dynamics and dynamicity; lack of commitment; trust building; conflict management and negotiation; risk identification and management; decision-making process slowness; increase the product/service value; other organizational, financial, technological issues etc. [1] $[7][12][26][28][30][33][41][44][45][46]$.

Business processes challenges comprise how organizations plan and design the partnership operation, such as alignment of heterogeneous organizations; more complex business process identification and design; outputs and business model redesign for performance improvement; decision-making and coordination processes establishment; responsibilities assignment; shared resources use etc. [7][12][26][33] [41][44][45]. 
Knowledge management challenges comprise how organizations capture, store, retrieve, share and use the available knowledge, such as organization and elicitation of a wide variety of information; capturing, organizing, sharing, reusing and applying several types of knowledge distributed in isolated bases; reducing differences of cultures, values, know-hows and processes; common ground establishment and maintaining; lack of information sharing; organizations heterogeneity leading to cultural and ontological differences; lack of supporting tools to manage distributed knowledge etc. [7][24][26][30][33].

Collaboration challenges comprise how participating organizations work together to achieve their mutual goal, such as inappropriate collaboration agreement; increasing cohesion between participating organizations; establishment of a sense of community; provision of infrastructure for collaboration and integration; skills development to work on a new work dynamics; risk of opportunism from partners; lack of communication etc. [12][26][28][33][41][45].

\subsection{ICT related researched areas}

Literature on cross-organizational management has investigated several ICT related areas, detailing authors' findings, the main challenges faced and open issues. Some authors argue that organizations' members are key elements for cross-organizational alignment since they act as agents of their organizations and serve as channels through which all organizational dynamics are shared with the partner organization [13][16]. When forming a mixed and multicultural context, these organization's members, who operated with never-before-asked assumptions, must collaborate, which evidence differences. Therefore, to ensure the joint work effectiveness, it is necessary to disseminate a collaborative culture and provide an enabling environment for collaboration, thus encouraging these members to be committed to mutual goals, be aware of differences and learn to behave in a new and different organizational dynamic [7][8] [16][32]. Proposals for defining organizations' collaborative profile [32], thus assisting the assessment of their readiness for collaboration; providing awareness of the adopted criteria to evaluate joint results, thus allowing to understand and assess how joint work evolves over time [26]; detailing collaboration patterns [25], i.e. a prescription which addresses a collaborative problem that may occur repeatedly in the environment; and helping to build a collaborative environment; are found in literature.

Enterprise Architecture is another aspect to be considered [3][4][14][47][48][49][50]. The way organizations establish their strategies of planning; de- cision-making; existing knowledge and skills identification, security and application; resource allocation; process, systems and information designing etc., differs from each other. With the need to work together, participating organizations should explain how to model and organize the information flow, facilitate the knowledge exchange and maintain intellectual property rights, so a cross-enterprise architecture can be built [7][12][51]. Thus, comprehending the existing architecture allows understanding how each organization can best contribute to the partnership, establish agreements to provide and use the available (physical and informational) resources and better integrate them through a strategic alignment.

Cross-organizational management also encompasses the creation of value from managing intangible actives of organizations through knowledge creational processes, sharing and utilization [9]. Thus, the joint work requires that organizations capture, organize, store, retrieve, share and use a wide variety of information, which are often distributed in isolated bases, to combine complementary internal and external knowledge [7][9][36][52]. With this, organizations can generate new relevant knowledge to the partnership and learn from each other [36][53]. Therefore, it is necessary to stimulate the combination of internal and external knowledge, since knowledge management does not often go beyond the organization's border, providing tools that reduce the gap between the variety of existing concepts and support all phases of knowledge management [36] [52]. Besides that, it is important that organizations understand that people know different things and know the same things in different ways, and recognize that the knowledge management and decisionmaking affect all participant organizations, so they all are responsible for and must be committed to it [24] [26]. The knowledge diversity is beneficial for joint work and must be appropriately managed.

It is also necessary to understand what the crossorganizational relationship expects to achieve and what can be actually done. Organizations develop and maintain a great collection of business process models to represent the complex system that they are, becoming necessary to capture and analyze these processes, their interdependencies with each other and existing service level agreements (SLAs) [37] [54]. Business and mutual goals modeling become more complex when dealing with several organizations interacting with each other. There is a need to integrate different processes, to standardize multiple concepts, to assign responsibilities and authorities, to establish performance indicators and to understand that definitions and changes impact not only one organization but the entire partnership [13][22][36] 
[55]. Methodologies for understanding an organization should be improved and extended to deal with cross-organizational relationship particularities, providing mechanisms that represent the collaboration aspects of the joint work, make visible and ready-to-use the combined knowledge, allow "plugand-play" adjustments and help a real-time management [14][15][56][57][58].

The management itself is another aspect discussed. Organizations need to organize, allocate and coordinate their resources, activities and dependencies to achieve the mutual goal within the required time, costs and quality frame [11]. The crossorganizational management involves (a) resource management [12][44], comprising strategies for managing shared personnel, controlling resources availability, defining resources priority access etc.; (b) change management, comprising strategies for identifying reasons for changing, types (process, control, collaboration, automation, actors etc.) and life cycle affected phases, engaging participants in change process, managing decision-making, problem solving, negotiation and collaboration between partners etc.; (c) risk management [43], comprising strategies for identifying and prioritizing existing risks; (d) performance management [23][25][26][36][59], comprising strategies for establishing performance indicators, evaluating each organization performance and the relationship performance, being aware of partner criteria to evaluate joint results etc.; besides other aspects considered while managing any project (time, cost, quality, communication, acquisition etc.).

Besides that, it is also necessary to think over systems adopted by organizations (Interorganizational Information Systems - IOIS). These systems must support the necessary activities for the partnership and be aligned with business strategy [60][61][62] [63][64][65][66]. Thus, issues as dynamic environment, undefined or nonexistent administrative structure, undefined locations, variable duration of the partnership, dynamic and variable organizations' borders, shared skills and knowledge, many involved people and from different organizations, information from multiple domains etc., will influence a crossorganizational systems development [30].

Another important issue is the trust between partners. Trust is defined as "one party's expectation that the other party can be relied on to fulfill obligations, behave in a predictable manner, and act and negotiate fairly even when the possibility for opportunism exists", implying that organizations allow for mutual vulnerability, risk and positive expectations [7][36]. It is dynamic, i.e. varies over time, and should be assessed according to the desired level (trust of each partner or trust of partnership as a whole) and consid- ering factors, such as organization reputation, information sharing, collaboration, established agreements, environmental uncertainty etc. [7][10][35][36] [67]. By perceiving a trusted cross-organizational relationship, organizations are more likely to collaborate, share information and integrate actions, thus leading to an increasingly transparent environment and a greater chance of achieving the mutual goal.

\section{Conclusion}

The need to remain competitive in a dynamic, unpredictable and challenging environment lead organizations to establish partnerships for combining resources, knowledge and power in benefit of participating organizations. However, it is a challenge to make these organizations being integrated and working in a new and different dynamic, thus ensuring the joint work will be effective and successful.

This paper has explored the cross-organizational relationship management. It was developed and performed an SLR to understand how literature has been facing collaboration and relationship establishment between different organizations, besides organizing an ICT body of knowledge about this topic.

The retrieved literature has allowed to better understand the cross-organizational relationship management, with studies investigating several ICT related areas and detailing approaches to help organizations in different aspects when engaging in partnerships with other organizations. However, besides a lot of work has been done, retrieved literature are only interested in some aspect of cross-organizational relationship management, with many issues still open for investigation. Concerning creation, we can cite issues as partners' identification and selection; and collaboration and operation planning (comprising the proper type of relationship and structure identification; more complex business process design; responsibilities assignment; risk identification). Regarding operation and evaluation, we can cite issues as the provision of infrastructure for collaboration and integration; managing distributed and heterogeneous knowledge; and organizational and crossorganizational performance indicators establishment and monitoring. As for dissolution, we can cite the definition of collaboration inheritance. Therefore, some mechanism that supports organizations to identify what is relevant and necessary for the partnership is still required. There are many factors to consider and little guidance is provided to make the crossorganizational relationship effective and successful. Further research on making organizations more integrated, prepared to interoperate their processes and information, and able to act and achieve mutual goals 
even with existing differences are necessary, with initial efforts of systematization being detailed in [2].

However, even identifying several benefits from the performed SLR, it is important to highlight some challenges that need to be overcome. The first challenge concerns the SLR coverage. The designed protocol has limited the search to studies published over the last decade. The inclusion of prior studies could provide additional knowledge about crossorganizational management, thus improving the obtained results. Another challenge is the topic extent. As detailed above, it is possible to focus on different areas to conduct research on cross-organizational relationships. Thus, it is imperative to carefully delimit what is or is not in the research project scope.

As future work, the designed SLR could be reexecuted, by researchers or other people, to improve the body of knowledge about the investigated topic. Besides that, an approach to support the crossorganizational relationship management is under development. In addition, we aim to conduct experiments for applying this approach in real scenarios to evaluate its impact and gather inputs that help its improvement and evolution.

\section{Acknowledgments}

The authors wish to thank CAPES by grants supporting the research.

\section{References}

[1] A-M. Croteau; J. Dubsky. "Uncovering Modes of Interorganizational Governance of IT". In: Hawaii International Conference on System Sciences, 2011.

[2] B. Diirr; C. Cappelli. "Establishing transparent interorganizational relationships through shared goals for anti-corruption in Brazil”. In: Business Process Management-BPMGOV Workshop, 2017.

[3] P. Drews; I. Schirmer. "From Enterprise Architecture to Business Ecosystem Architecture". In: International Enterprise Distributed Object Computing Conference, pp. 13-22, 2014.

[4] T. Mueller; D. Schuldt; B. Sewald; M. Morisse; J. Petrikina. "Towards inter-organizational Enterprise Architecture Management”. In: Americas Conference on Information Systems, 2013.

[5] H. Ranaei; A. Zareei; F. Alikhani. "Interorganizational Relationship Management: A Theoretical Model". International Bulletin of Business Administration, n. 9, pp. 20-30, 2010.

[6] F. Saadatmand; R. Lindgren. "The Tension between Stabilized Cooperation and Intensified Competition: Greening of Technological Frames in Practice". In:
Hawaii International Conference on System Sciences, pp. 327-336, 2016.

[7] P. Van Fenema; B. Keers; H. Zijm. "Interorganizational Shared Services: Creating Value across Organizational Boundaries". Shared Services as a New Organizational Form, v. 13, pp. 175-217, 2014.

[8] J. Harrison. Strategic Management of Resources and Relationships, Wiley, 2003.

[9] A. Lisboa-Soh; N. Casarotto-Filho; I. José-Cunha. "Interorganizational Knowledge Management". Dirección y Organización, v. 48, pp. 34-40, 2012.

[10] J. Mun; M. Shin; M. Jung. "A goal-oriented trust model for virtual organization creation". Journal of Intelligent Manufacturing, v. 22, pp. 345-354, 2011.

[11] W. Obidallah; B. Raahemi; F. Alaieri. "Change processes and procedures in service oriented virtual organizations and collaborative network". In: International Conference on Management of Emergent Digital EcoSystems, pp. 50-55, 2014.

[12] A. Shamsuzzoha; C. Tosano; L. Carneiro; V. Kumar; P. Helo. "ICT-based solution approach for collaborative delivery of customized products". Production Planning \& Control, v. 27, n. 4, pp. 280-298, 2016.

[13] J. Bocanegra; J. Pena; A. Ruiz-Cortes. "Interorganizational Business Modeling: An Approach for Traceability of Goals, Organizational Models and Business Processes". IEEE Latin America Transactions, v. 9, n. 1, pp.847-854, 2011.

[14] C. Legner; K. Wende. "The Challenges of Interorganizational Business Process Design - a Research Agenda". European Conference on Information Systems. 2007. Available at: $<$ http://works.bepress.com/hubert_oesterle/172/>.

[15] M. Sebu; H. Ciocârlie "Merging business processes for a common workflow in an organizational collaborative scenario". In: International Conference on System Theory, Control and Computing, pp. 134-139, 2015.

[16] Z. Zhu; H. Huang. "The Cultural Integration in the Process of Cross-border Mergers and Acquisitions". International Management Review, v. 3, n. 2, pp. 4044, 2007.

[17] B. Kitchenham. "Procedures for Performing Systematic Reviews". Technical Report TR/SE-0401, Keele University, 2004

[18] M. Kuhrmann; D. M. Fernández; M. Daneva. “On the pragmatic design of literature studies in software engineering: An experience-based guideline". Empirical Software Engineering, 2017.

[19] M. Niazi. "Do Systematic Literature Reviews Outperform Informal Literature Reviews in the Software Engineering Domain? An Initial Case Study". Arabian Journal for Science and Engineering, v. 40, n. 3, pp. 845-855, 2015. 
[20] J. Biolchini; P.G. Mian; A.C. Natali; G.H. Travassos. "Systematic Review in Software Engineering: Relevance and Utility". Technical Report ES67905, PESC-COPPE/UFRJ, 2005.

[21] M. Pai; M. Mcculloch; J. Gorman; N. Pai; W. Enanoria; G. Kennedy; P. Tharyan; J. Colford. "Systematic reviews and meta-analyses: An illustrated, step-bystep guide". The National Medical Journal of India, v. 17, n. 2, pp. 86-95, 2004.

[22] M.H. Danesh; B. Raahemi; S.M.A. Kamali; G. Richards. "A distributed service oriented infrastructure for business process management in Virtual Organizations". In: IEEE Canadian Conference on Electrical and Computer Engineering, pp. 1-6, 2012.

[23] S. Kang; T. Moon. "Supply Chain Integration and Collaboration for improving Supply Chain Performance: A Dynamic Capability Theory Perspective". In: Hawaii International Conference on System Sciences, pp. 307-316, 2016.

[24] R. Swarnkar; A. Choudhary; J. Harding; B. Das; R. Young. "A framework for collaboration moderator services to support knowledge based collaboration". Journal of Intelligent Manufacturing, v. 23, pp. 20032023, 2012.

[25] Y. Verginadis; D. Apostolou; N. Papageorgiou; G. Mentzas. "Collaboration patterns in event-driven environments for virtual organizations". AAAI Spring Symposium - Technical Report SS-09-05, 2009.

[26] J.M. Carroll; J. Wang. "Designing Effective Virtual Organizations as Sociotechnical Systems". In: $\mathrm{Ha}$ waii International Conference on System Sciences, pp. 1-10, 2011.

[27] L. Lewis; M. Isbell; M. Koschmann. "Collaborative tensions: Practitioners' experiences of interorganizational relationships". Communication Monographs, v. 77, pp. 460-479, 2010

[28] A. Samdantsoodol; S. Cang; H. Yu. "Overview of virtual enterprises in supply chain management". In: International Conference on Automation and Computing: Integration of Design and Engineering, pp. 193201,2012.

[29] J. Sousa; J. Branco; B. Amorim. "Modeling Information Systems Sociomateriality and its Impact on Organizational Cooperative Environment Development". Procedia Computer Science, v. 64, pp. 11401148,2015

[30] L. Priego-Roche; A. Front; D. Rieu. "A framework for virtual organization requirements". Requirements Engineering, v. 21, pp. 439-460, 2016.

[31] G. Johnson; R. Whittington; K. Scholes. Exploring corporate strategy, Prentice Hall, 2008.

[32] D. Romero; N. Galeano; A. Molina. "Mechanisms for assessing and enhancing organisations' readiness for collaboration in collaborative networks". Internation- al Journal of Production Research, v. 47, pp. 46914710,2009

[33] H. Afsarmanesh; L. Camarinha-Matos. "On the classification and management of Virtual organisation Breeding Environments". International Journal of Information Technology and Management, v. 8, pp. 234-259, 2009

[34] M.H. Danesh; B. Raahemi; M.A. Kamali. "A framework for process management in service oriented virtual organizations". In: International Conference on Next Generation Web Services Practices, pp. 12-17, 2011.

[35] K. Mirkovski; P. Lowry; B. Feng. "Factors that influence interorganizational use of information and communications technology in relationship-based supply chains: Evidence from the Macedonian and American wine industries". Supply Chain Management, v. 21, pp. 334-351, 2016.

[36] B. Sun; Y-J. Lo. "Achieving alliance ambidexterity through managing paradoxes of cooperation: A new theoretical framework". European Journal of Innovation Management, v. 17, pp. 144-165, 2014.

[37] A. Del-Río-Ortega; A.M. Gutiérrez; A. Durán; M. Resinas; A. Ruiz-Cortés. "Modelling Service Level Agreements for Business Process Outsourcing Services". In: International Conference on Advanced Information Systems Engineering, LNCS 9097, pp. 485-500, 2015

[38] A. Del-Río-Ortega; M. Resinas; A. Durán; A. RuizCortés; M. Toroa. "VISUAL PPINOT: A Graphical Notation for Process Performance Indicators". Decision Support Systems, 2015.

[39] M. Khalfallah; N. Figay; P. Ghodous; C.F. Da Silva. "Cross-Organizational Business Processes Modeling Using Design-by-Contract Approach”. In: International IFIP Working Conference on Enterprise Interoperability, LNBIP 144, pp. 77-90, 2013.

[40] M. Arasteh; M. Amini; R. Jalili. "A trust and reputation-based access control model for virtual organizations". In: International Conference on Information Security and Cryptology, pp. 121-127, 2012.

[41] M. Cancian; R. Rabelo; C. von Wangenheim. "Supporting processes for collaborative SaaS". IFIP Advances in Information and Communication Technology, v. 408, pp. 183-190, 2013.

[42] T. Dimitrakos; D. Golby; P. Kearney. "Towards a Trust and Contract Management Framework for Dynamic Virtual Organizations”, In: eChallenges, 2004.

[43] M. Alawamleh; K. Popplewell. "Interpretive structural modelling of risk sources in a virtual organization". International Journal of Production Research, v. 49, pp. 6041-6063, 2011.

[44] A. Gueye; D. Moussavou; H. Saliah-Hassane; S Ouya; I. Sanogo; C. Lishou. "Dynamic publishing 
and availability management of virtual machines in Virtual Organization". In: Global Engineering Education Conference, pp. 793-798, 2015.

[45] A. Luo; M. Murphy; T. Hanss. "Human infrastructure as process and effect: Its impact on individual scientists' participation in international collaboration". In: International Conference on Support Group Work, pp. 45-53, 2012.

[46] K. Riemer; N. Vehring. "Virtual or vague? A literature review exposing conceptual differences in defining virtual organizations in IS research". Electronic Markets, v. 22, pp. 267-282, 2012.

[47] T. Choi; I. Kröschel. "Challenges of governing interorganizational relationships: Insights from a case study". In: European Conference on Information Systems, pp. 1-16, 2015.

[48] J. Köpke; J. Eder; M. Künstner. "Projections of Abstract Interorganizational Business Processes". In: International Conference on Database and Expert Systems Applications, LNCS 8645, pp. 472-479, 2014.

[49] A. Montarnal; T. Wang; S. Truptil; F. Bénaben; M. Lauras; J. Lamothe. "A Social Platform for Knowledge Gathering and Exploitation, Towards the Deduction of Inter-enterprise Collaborations". Procedia Computer Science, v. 60, pp. 438-447, 2015.

[50] A. Oberweis; T. Schuster. "A meta-model based approach to the description of resources and skills". In: Americas Conference on Information Systems, pp. $3677-3688,2010$

[51] M. Bakhtiyari; A. Barros; N. Russell. "Enterprise architecture for business networks: A constructivist synthesis". In: Australasian Conference on Information Systems, 2014.

[52] D. Langenberg; C. Kind; M. Dames. "Knowledge management in cloud environments". In: International Conference Proceeding Series, 2011.

[53] S. Choi; I. Ko. "The Effects of Electronic Collaboration on Interorganizational Learning and Firm Performance". In: Hawaii International Conference on System Sciences, 2010.

[54] R-H. Eid-Sabbagh; R. Dijkman; M. Weske. "Business Process Architecture: Use and Correctness". In: International Conference on Business Process Management, LNCS 7481, pp. 65-81, 2012.

[55] D. Knuplesch; M. Reichert; J. Mangler; S. RinderleMa; W. Fdhila. "Towards Compliance of CrossOrganizational Processes and their Changes? - Research Challenges and State of Research". In: International Conference on Business Process Management, LNBIP 132, pp. 649-661, 2012.

[56] K. Bouchbout; A. Alimazighi. "Inter-Organizational Business Processes Modelling Framework". In: Conference on Advances in Databases and Information Systems, 2011.
[57] A. Lawall; T. Schaller; D. Reichelt. "Restricted Relations between Organizations for Cross-Organizational Processes". In: Conference on Business Informatics, 2014.

[58] D. Lin; T. Ishida. "Coordination of Local Process Views in Interorganizational Business Process". IEICE Transactions on Information and Systems, v. E97-D, n. 5, pp. 1119-1126, 2014

[59] I. Westphal; K-D. Thoben; M. Seifert. "Managing collaboration performance to govern Virtual Organizations". Journal of Intelligent Manufacturing, v. 21, pp. 311-320, 2010 .

[60] N. Frick; T.F. Küttner; P. Schubert. "Assessment Methodology for a Maturity Model for Interorganizational Systems: The Search for an Assessment Procedure". In: Hawaii International Conference on System Sciences, pp. 274-283, 2013.

[61] C. Hsu; Y-T. Lin; T. Wang. "A legitimacy challenge of a cross-cultural interorganizational information system". European Journal of Information Systems, v. 24 , pp. $278-294,2015$.

[62] J. Kauremaa; K. Tanskanen. "Designing interorganizational information systems for supply chain integration: a framework". The International Journal of Logistics Management, v. 27, n. 1, pp. 71-94, 2016.

[63] M. Mandrella; S. Zander; L. Kolbe. "IT-Based Value Co-Creation: A Literature Review and Directions for Future Research". In: Hawaii International Conference on System Sciences, pp. 287-296, 2016.

[64] T. Phuaphanthong; T. Bui. "How Did It Happen? A Memetic Theory of Interorganizational Information Systems Emergence and Evolution". In: Hawaii International Conference on System Sciences, pp. 45264535,2014

[65] K. Sun; W.C. Lai. "ISAM-based inter-organization information systems alignment process". In: International Conference on Computer Science and Service System, pp. 1358-1361, 2011.

[66] K. Sun; K. Yu. "Research on project management for Inter-Organizational Information Systems". In: International Conference on E-Business and $E$ Government, pp. 1-4, 2011.

[67] M. Daudi; S. Msanjila. "Modeling of evolution and sustainability of rational trust in dynamic Vos". IFIP Advances in Information and Communication Technology, v. 408, pp. 548-555, 2013 